\title{
Cytoreductive surgery with hyperthermic thoracoabdominal chemotherapy in stage IV ovarian cancer - a technical description
}

\author{
Mukur Dipi Ray ${ }^{1}$, Raghuram Kuppusamy¹, Navin Kumar ${ }^{1}$, Rakesh Garg² \\ 'Department of Surgical Oncology, Dr BRA-IRCH, AlIMS, New Delhi 110029, India. \\ ${ }^{2}$ Department of Onco-anesthesiology and Palliative Medicine, Dr Dr. B.R.A Institute-Rotary Cancer Hospital, AlIMS, New Delhi \\ 110029 , India.
}

Correspondence to: Dr. Mukur Dipi Ray, MS, FRCS, PhD, Scholar, Department of surgical oncology, Dr BRA-IRCH, AlIMS, Ansari Nagar, New Delhi 110049, India. E-mail: dr_mdray@yahoo.com; Navin Kumar, MS, MCh Trainee, Department of surgical oncology, Dr BRA-IRCH, AIIMS, Ansari Nagar, New Delhi 110049, India. E-mail: navin2k1@gmail.com

\begin{abstract}
How to cite this article: Ray MD, Kuppusamy R, Kumar N, Garg R. Cytoreductive surgery with hyperthermic thoracoabdominal chemotherapy in stage IV ovarian cancer - a technical description. J Cancer Metastasis Treat 2020;6:9. http://dx.doi.org/10.20517/2394-4722.2020.16
\end{abstract}

Received: 18 Feb 2020 First Decision: 23 Mar 2020 Revised: 26 Mar 2020 Accepted: 3 Apr 2020 Published: 17 Apr 2020

Science Editor: Pravin D. Potdar Copy Editor: Jing-Wen Zhang Production Editor: Tian Zhang

\begin{abstract}
Systemic chemotherapy for peritoneal disease in ovarian carcinoma is associated with a recurrence rate of more than $75 \%$, and most of the cases are confined to the peritoneal cavity. The propensity of locoregional treatment failure has paved the way for the discovery of cytoreductive surgery with intra-cavitary chemotherapy. Cytoreductive surgery (CRS) is the present-day treatment modality for a variety of peritoneal carcinomatosis including ovarian cancer, and multi-visceral resection is critical for completion of CRS. In cases of diaphragmatic infiltration by tumor deposits, partial resection leads to a diaphragmatic rent, which can be used for the perfusion of chemotherapeutic drugs into the pleural cavity. Disease transmission from the peritoneal to pleural cavity is a poor prognostic factor however. Hence, intrathoracic hyperthermic chemotherapy may be a reasonable treatment option for ovarian carcinoma with malignant pleural effusion or pleural deposits. Hyperthermic intraperitoneal chemotherapy (HIPEC) is added to the treatment plan in cases of complete CRS but this is a technically demanding procedure. Therefore, performing hyperthermic intrathoracic chemotherapy on top of CRS and HIPEC may be even more complicated for such advanced cancers. The technique of combining HIPEC and hyperthermic intrathoracic chemotherapy is also commonly known as hyperthermic thoracoabdominal chemotherapy (HITAC). The perioperative morbidity and mortality may be remarkably high in such scenarios. We describe our CRS technique with HITAC, which was performed in three FIGO stage IVA ovarian carcinoma patients with metastatic pleural effusion after complete CRS. The patients were retrospectively identified from a prospectively maintained database. All had partial diaphragmatic resection followed by HITAC as part of CRS treatment. Surgical techniques
\end{abstract}

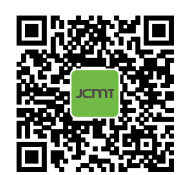


are outlined along with accompanying intra-operative images. Patient demographics, clinical and follow-up details were also described briefly. No comparative analysis with control patients was done. Adjustments in chemotherapy dose are not mandatory for HITAC. Of three patients, one had intrathoracic recurrence on followup; no mortality was recorded HITAC is a complex and potentially harmful procedure whose toxicity profile is still poorly known. Morbidity was not life-threatening and survival was acceptable.

Keywords: Hyperthermic intraperitoneal chemotherapy, hyperthermic intrathoracic chemotherapy, ovarian carcinoma, cytoreduction surgery, peritoneal carcinoma index

\section{INTRODUCTION}

Previously, peritoneal disease was considered terminal and systemic chemotherapy was offered for palliative intent while palliative surgery only had a role in symptom relief. With the advent of cytoreductive surgery (CRS) in the early 1990's, it is now the accepted treatment modality for a subset of patients with peritoneal carcinomatosis from pseudomyxoma peritonei, appendiceal adenocarcinoma and mesothelioma, and has also showed promising results in selected patients with ovarian, colorectal and gastric cancer ${ }^{[1]}$. The purpose of CRS is to resect all macroscopic disease through peritonectomy and involved viscera followed by intraperitoneal chemotherapy by targeting residual microscopic disease through provision of a high intraperitoneal concentration with lower systemic toxicity ${ }^{[2]}$. If CRS is complete, the more provocative procedure hyperthermic intraperitoneal chemotherapy (HIPEC) is performed in the same setting ${ }^{[2,3]}$. HIPEC improves both quality of life and survival ${ }^{[4]}$. These treatments are based on the concept that when disease is limited to the peritoneal cavity, it is still considered locoregional. The comprehensive CRS approach was described by Dr. Sugarbaker ${ }^{[3]}$ in 2007. Evidence for hyperthermia is based on accelerated cell death at $41-43{ }^{\circ} \mathrm{C}$ in experimental settings ${ }^{[5]}$. The additive toxic effects of HIPEC have also been documented in the literature ${ }^{[6]}$. Interval CRS and HIPEC resulted in longer recurrence-free and overall survival among FIGO stage III epithelial ovarian cancer than surgery alone, and did not result in excessive side effects ${ }^{[7]}$. It also offers a significant survival benefit to patients with recurrent epithelial ovarian cancer, especially in patients with complete $\mathrm{CRS}^{[8]}$. There is no randomized controlled study or feasibility study demonstrating the efficacy of hyperthermic thoracoabdominal chemotherapy (HITAC) over HIPEC however. Erasmus et al. ${ }^{[9]}$ reported that chemotherapeutic drugs were also absorbed from the pleural cavity like the peritoneal cavity. In the case of HITAC, the intrapleural concentration of chemotherapeutic drugs was persistently high compared to plasma. The current study is focused on the technical aspects and feasibility of HIPEC and HITAC in ovarian cancer patients. It does compare HIPEC alone with HIPEC and HITAC. Cognizant of the beneficial effects of HIPEC in selected patients with ovarian cancer, the same strategy was applied through HITAC in patients with thoracic involvement. This is the first study of its kind in the Indian patient population.

\section{MATERIALS AND METHODS}

This is a retrospective study of three prospectively selected patients with ovarian carcinoma and metastatic pleural effusion treated with CRS and HITAC after neoadjuvant chemotherapy. The aim was to describe the technical aspects of the surgery with brief descriptions of the postoperative outcomes and treatmentrelated morbidities on follow up.

\section{CRS technical aspects}

For CRS, a midline laparotomy extending from the xiphoid process to symphysis pubis was performed to provide greater exposure of the abdomen. Bilateral pelvic and retroperitoneal lymph node dissection with total omentectomy were done routinely as a part of CRS in ovarian cancer apart from total hysterectomy and salpingo-oophorectomy. Regarding peritonectomy, we do not routinely practice total peritonectomy 


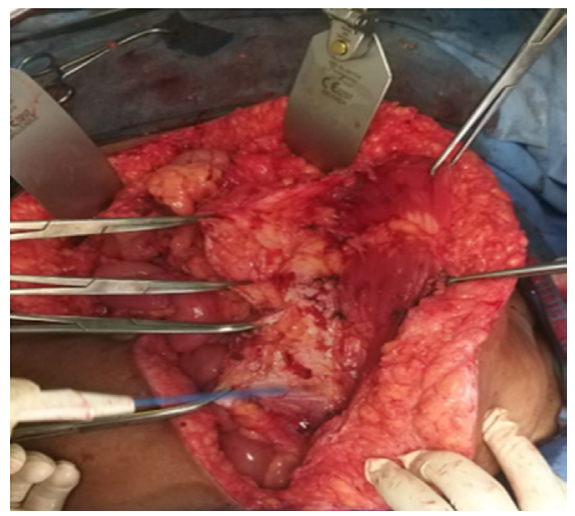

Figure 1. Peritonectomy using multiple artery forceps for retraction

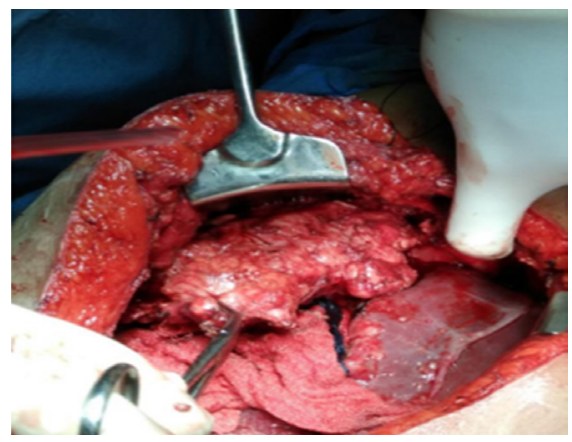

Figure 2. Diaphragmatic peritonectomy with full-thickness resection of diaphragmatic deposits

in all cases. Selective peritonectomy was performed in the region(s) macroscopically affected by the tumor. Total peritonectomy was performed in two cases in the present study, which had gross peritoneal disease. Total peritoneal stripping in continuity is a technically demanding procedure, hence in the current study, we followed the split technique, which involves stripping and removal of the entire peritoneum in five parts - right subdiaphragmatic peritoneum along with Glisson's capsule, left sub-diaphragmatic peritoneum, right and left parietal wall/paracolic gutter peritoneum and pelvic peritoneum. Peritonectomy was performed by holding and lifting the peritoneal edges with multiple artery forceps [Figure 1].

Surgical dissection was performed using monopolar diathermy with a sharp tip and diathermy settings at 30 coagulation spray mode although many surgeons prefer ball tip diathermy in pure cut mode. Additional visceral organ resection (colectomy, colo-proctectomy, splenectomy, gastrectomy, appendicectomy, cholecystectomy, liver resection and small bowel resection) may be performed, depending upon involvement. Whenever bowel resection is required, we prefer resection-anastomosis before HIPEC. Bowel edema, erythema and other hyperthermic chemotherapy-induced changes due to HIPEC at the edges of the bowel wall may become a constant threat for anastomotic leaks. For diaphragmatic peritonectomy, access and exposure of the diaphragmatic peritoneum were of utmost importance. Adequate exposure was obtained with the Omni-Tract surgical retractor and the liver was completely mobilized, except at the area of the hepatic veins and the suprahepatic inferior vena cava. For full-thickness large solid deposits involving the diaphragm, we performed full thickness diaphragmatic resection in two cases of the present study. In another case, partial resection of the hemidiaphragm was created for HITAC. Full-thickness resection of the diaphragm and the subsequent diaphragmatic rent created are shown in Figure 2. Pleural nodules were excised through the same rent in one case. The other two cases had only pleural effusion with no pre-operative or intra-operative evidence of metastatic pleural deposits. Total parietal pleurectomy was 


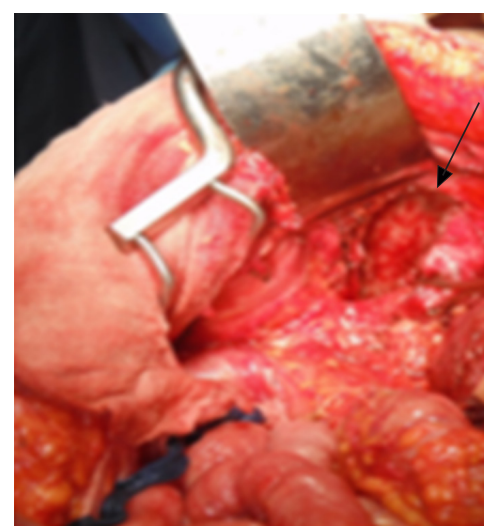

Figure 3. Diaphragmatic rent after partial diaphragmatic excision (black arrow)

not performed. We aspirated the pleural effusion in each case and dissected a few pleural deposits with electro-diathermy in one case. Thorough pleural lavage with chemotherapeutic perfusate into the pleural cavity was performed during HIPEC.

\section{The technique of diaphragmatic resection}

For diaphragmatic resection, one must be aware of the anatomy of the diaphragm in relation to the phrenic nerve. The phrenic nerve originates mainly from the 4 th cervical nerve, but also receives contributions from the 5 th and 3 rd cervical nerves (C3-C5).

The right phrenic nerve enters the diaphragm through the central tendon or inferior vena cava opening. On the right side, it courses relatively more medially throughout its thoracic course to various structures like the right brachiocephalic vein, SVC and pericardium over the right atrium. The inferior vena cava lies medially and reaches under the surface of the diaphragm by passing through the inferior vena cava foramen in the central tendon.

The left phrenic nerve pierces the superior surface of the muscular part of the diaphragm, just to the left border of the heart.

Both nerves divide or trifurcate at, or just above the diaphragm. The branches travel together into the diaphragmatic musculature, while small sensory branches supply the peritoneum over the central part of the diaphragm. The larger motor branches separate within the diaphragm into four major nerves trunks sternal, anterolateral, postero-lateral and crural. The nerve trunks travel partly within the diaphragmatic muscle and innervate the inferior surface covered by peritoneum. Therefore, the diaphragmatic incision has to be made circumferentially to avoid the main phrenic nerve trunks.

In the present study, we made an incision in the above-mentioned manner and excised the tumor deposits.

The patient's head end was lowered during HITAC procedures, so that the chemotherapeutic fluid can gain easy access to the thoracic cavity by free flow from the abdominal cavity.

\section{Reconstruction of the diaphragm after HITAC procedure}

In two cases, we had to incise approximately one-fourth of the diaphragm [Figure 3]. The diaphragmatic defects were repaired primarily with polypropylene 1-0 suture. In another case, the peritoneal defect was almost 50\% and required reconstruction with polypropylene mesh, fixed with polypropylene 1-0 suture. In all three cases, we placed the ICD in the triangle of safety. 


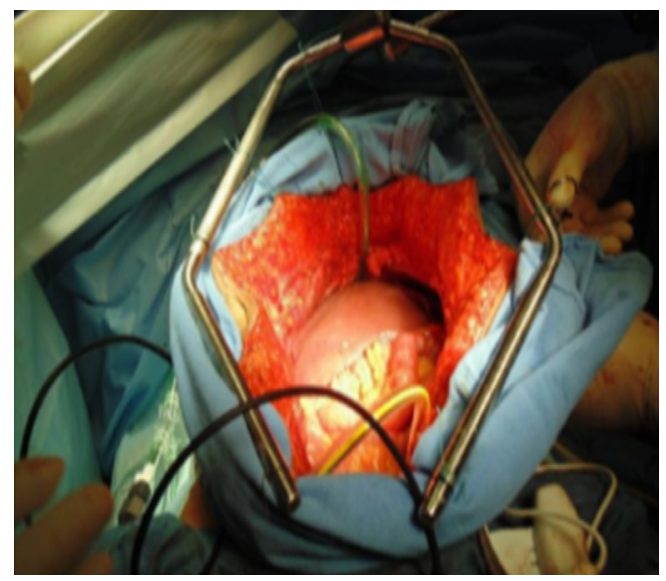

Figure 4. Demonstrating the creation of coliseum for performing hyperthermic intraperitoneal chemotherapy

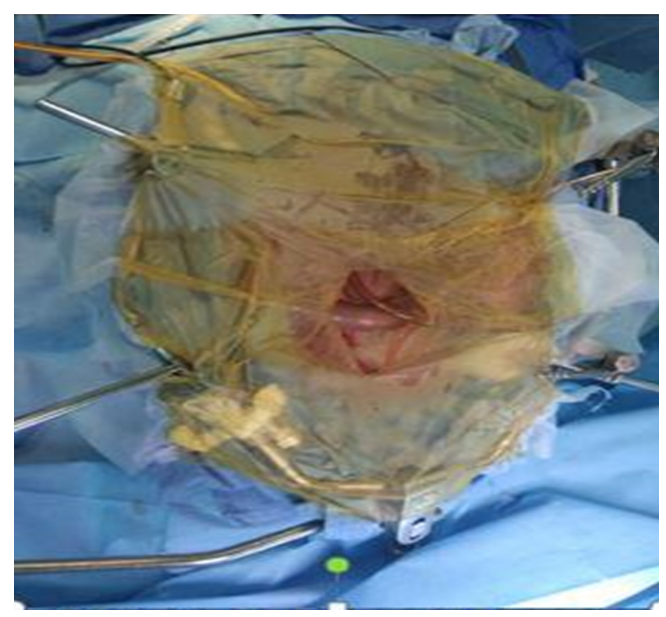

Figure 5. Showing the coliseum (semi-open technique) and hyperthermic intraperitoneal chemotherapy tubes with adhesive sheet

\section{HITAC: technical aspects}

HIPEC was performed using the open Coliseum technique [Figure 4] as described by Sugarbaker ${ }^{[3]}$. We used the Omni-Tract surgical retractor for exposing the abdominal cavity. Skin edges were suspended using interrupted polyester sutures fixed to the horizontal arms of the retractor to create an open space in the abdominal cavity, as depicted in Figure 4. An avascular plane of about $2-3 \mathrm{~cm}$ was routinely created between the anterior rectus sheath and subcutaneous tissue so that the rectus sheath and muscle would be immersed in the HIPEC perfusate.

An adhesive plastic sheet was incorporated to prevent spillage of the chemotherapy solution and heat loss [Figure 5]. A slit was made in the plastic cover to allow manual access to the abdomen and pelvis during HITAC. This helped in cases of blocked tubings during HIPEC and was also used to ensure uniform distribution of the perfusate and heat by constant, intermittent manipulation of the perfusate. A specially designed wooden spatula was used for stirring the heated chemotherapy solution to ensure uniform distribution as well as heat. Elbow-length gloves were worn for manual stirring and debris removal from the HIPEC tubing ports.

In cases of ovarian carcinoma, cisplatin was used as a chemotherapeutic drug in HITAC. Apart from routine drug dosage calculation using body surface area, another method of estimation uses the 
approximate volume of the peritoneal cavity in litres. Dosage was calculated at $50 \mathrm{mg}$ of cisplatin per litre. The chemotherapeutic solution was prepared by a resident doctor/specialized staff nurse using aseptic technique and full body personal protective equipment in a separate room adjacent to the surgical suite. The desired temperature from the inflow tube was kept in the range between $42{ }^{\circ} \mathrm{C}$ to $44{ }^{\circ} \mathrm{C}$ and out-flow was maintained at $41{ }^{\circ} \mathrm{C}$ to $43{ }^{\circ} \mathrm{C}$. Per the literature, microscopic as well as tumour deposits up to $2.5 \mathrm{~mm}$ are destroyed by the synergistic effects of hyperthermia $\left(42^{\circ} \mathrm{C}\right)$ and chemotherapy ${ }^{[5]}$. To achieve the desired temperature in the abdominal cavity, the temperature in the heat exchanger of the machine was regularly titrated depending on the recorded temperature. During combined HIPEC and HITAC for 60 min with close intra-operative monitoring of core body temperature, hemostasis was ensured before starting. We also constantly monitored the flow rate and nature of fluid content in the tubings. The inflow tube was kept in the pelvis away from critical areas and the great vessels. The average perfusate volume was $2.30 \mathrm{~L}$, depending upon the capacity of the abdominal cavity. We used a triple-layered filtration mask, tightly fit to the face. It was also desirable to have a surgical smoke evacuator work continuously under the plastic sheet during perfusion and we used this in a number of cases. After completion of the procedure, the perfusate was aspirated, hemostasis ensured, and lung expansion confirmed before closure of the pleural defect. We did not perform peritoneal saline irrigation after completion of HITAC although some surgeons prefer to do so after clearing out the chemotherapeutic solution at the end of the procedure. Ipsilateral single intercostal drainage tube would then be placed. Two soft abdominal drains, one each in the sub-hepatic region and pelvis, were routinely placed in all cases. Additionally, we also placed a suction drain in the subcutaneous cavity after mobilizing the rectus sheath as mentioned earlier and this was removed on day 3 routinely unless drainage was more than $100 \mathrm{~mL}$ in the last $24 \mathrm{~h}$.

\section{Intraoperative monitoring}

The role of the anesthesiologist is crucial during CRS with HITAC because of the extensive resection and long duration of procedures. The addition of hyperthermia in HITAC presents further challenges for the clinicians so a team approach is paramount. The main concern is related to the various physiological changes that can occur during CRS with HITACe, where hyperthermia and the use of chemotherapeutic agents concurrently may affect body systems. These concerns relate mainly to major fluid shifts, respiratory, hemodynamic, renal, hepatic, hematological and metabolic changes along with electrolyte, fluid and thermal imbalances. The maintenance of normal physiology remains the main goal. Ventilatory strategies are also of a major concern, not only because of abdominal surgery but also from exposure of the thorax to chemotherapy drugs and hence, the need for single lung ventilation.

Preoperative assessment and optimization are thus required for optimal outcomes. A thorough history and examination is key and includes routine assessment along with evaluation of prior drug therapy including chemotherapy, analgesics or drugs for associated comorbidities. Preoperative rehabilitation is also emerging as an important management tool because of its various beneficial aspects in enhanced recovery after surgery.

Appropriate monitoring is essential for patients undergoing CRS and HITAC. Apart from routine conventional intra-operative monitoring (electrocardiogram, non-invasive blood pressure monitoring, pulse oximeter, capnography, temperature), certain additional monitoring strategies are required for such interventions. For airway management, the conventional endotracheal tube is used routinely with oropharyngeal core body temperature probe monitoring. However, single lung ventilation is desirable in cases of pleural deposit excision. Anesthesia induction is usually done using propofol, fentanyl and atracurium and maintained with atracurium, fentanyl and inhalational agents like sevoflurane or desflurane in oxygen and air mixture. Based on the extent of the abdominal mass and the patient's clinical condition, ventilator strategies may require further planning. 
Table 1. Showing the demographic and clinical details with follow-up status

\begin{tabular}{|c|c|c|c|}
\hline & Patient SI. No 1 & Patient SI. No 2 & Patient SI. No 3 \\
\hline Age (years) & 45 & 46 & 29 \\
\hline Date of registration & $08-05-2016$ & $04-06-2018$ & $27-06-2017$ \\
\hline Prior surgery & Staging laparotomy & None & None \\
\hline FIGO stage & IVA & IVA & IVA \\
\hline NACT (cycles/regimen) & 6\#TP, 6\#Gem + CDDP, 1\#TP & 12\#TP & 3\#TP \\
\hline Date of surgery & $20-05-2019$ & $08-11-2018$ & $12-03-2018$ \\
\hline Types of CRS & Secondary CRS & Interval CRS & Interval CRS \\
\hline CRS procedure & $\begin{array}{l}\text { Disease limited peritonectomy + omental } \\
\text { cake excision + terminal ileum and limited } \\
\text { right colon resection anastomosis }\end{array}$ & $\begin{array}{l}\text { TAH + BSO + B/L PLND + RPLND } \\
+ \text { total omentectomy + pouch of } \\
\text { douglas and liver deposit excision }\end{array}$ & $\begin{array}{l}\text { TAH + BSO + B/L PLND + RPLND + total } \\
\text { omentectomy + right diaphragmatic } \\
\text { stripping + selective peritonectomy }\end{array}$ \\
\hline CRS duration (min) & 370 & 410 & 330 \\
\hline Blood loss $(\mathrm{mL})$ & 1150 & 600 & 450 \\
\hline $\mathrm{PCl}$ & $23 / 39$ & $2 / 39$ & $15 / 39$ \\
\hline HITAC drug & Mitomycin & Cisplatin & Cisplatin \\
\hline Drug dosage (mg) & 30 & 100 & 100 \\
\hline Duration (min) & 60 & 45 & 60 \\
\hline Temperature $\left({ }^{\circ} \mathrm{C}\right)$ & 42 & 42 & 42 \\
\hline Perfusate & Normal saline & Normal saline & Normal saline \\
\hline Perfusate volume (L) & 2.5 & 3.0 & 2.5 \\
\hline CC score & 1 & 0 & 0 \\
\hline Comorbidity & Cl. Dindo II & Cl. Dindo III & Cl. Dindo II \\
\hline Adj chemotherapy & 3\#TP & $6 \# T P+B e v$ & 3\#TP \\
\hline Follow up status & Alive \& disease-free & Alive with disease & Alive \& disease-free \\
\hline
\end{tabular}

SI. No: serial number; FIGO: International Federation of Gynecology and Obstetrics; NACT: neoadjuvant chemotherapy; TP: Paclitaxel/ Carboplatin; CDDP: Cisplatin; TAH: total abdominal hysterectomy; BSO: bilateral salpingo-oophrectomy; B/L PLND: bilateral pelvic lymph node dissection; PAND: paraaortic node dissection; CRS: cytoreduction surgery; PCI: peritoneal carcinomatous index; HITAC: hyperthermic thoracoabdominal chemotherapy; CC score: completeness of cytoreduction score; Cl. Dindo: Clavien Dindo score; Adj: adjuvant; Bev: Bevacizumab

Goal-directed fluid therapy is desirable for fluid management. Monitoring for fluid management is routinely done using urine output measurement and non-invasive methods such as cardiac output monitors for assessing the fluid status to guide management. Multimodal analgesia is required for optimal outcomes. The use of thoracic epidural analgesia with local anesthetic and opioids appears to be acceptable in the current study. Coagulopathy needs to be identified and corrected as necessary. The use of point of care tools for assessing coagulopathy remains promising. Postoperative monitoring is crucial as these patients continue to have various physiological changes for days in the postoperative period. The patient should be monitored closely for fluid balance, hemodynamic fluctuations, renal impairment, coagulopathy and electrolyte imbalances. Such patients also need DVT prophylaxis in the postoperative period using pharmacological and/or mechanical measures.

\section{RESULTS}

CRS with HIPEC and HITAC were performed in three patients with ovarian carcinoma and peritoneal carcinomatosis after neoadjuvant chemotherapy.

Patients were 29-46 years with a mean age of 40 years. All patients resided in urban localities and were of middle class socioeconomic status.

Patient serial number "1" is a 45 -year-old female with known hypothyroidism. She underwent staging laparotomy at another institution for ovarian malignancy in December 2015 and histopathology was FIGO stage IB. The patient did not undergo any adjuvant chemotherapy. Six months later, she self-referred to our tertiary centre in May 2016 with symptoms of cough, breathlessness and abdominal distension and was diagnosed as FIGO stage IVA, recurrent ovarian carcinoma. The patient underwent chemotherapy with 
6 cycles of TP (Paclitaxel/Carboplatin), 6 cycles of Gem/CDDP (Gemcitabine/Cisplatin) and 1 cycle of TP (Paclitaxel/Carboplatin). Post-chemotherapy, the patient had partial response and she proceeded with secondary CRS and HITAC as mentioned in Table 1 . In the early postoperative period, the patient did not develop significant surgical morbidity (Clavien Dindo grade III/IV). Following three cycles of adjuvant chemotherapy, the patient remains disease-free with the last follow-up on 12-02-2020.

Patient serial number " 2 " is a 46 -year-old female with no known comorbidities nor significant family history. She initially presented to another institution with symptoms of abdominal pain, constipation, fever and weight loss. She was diagnosed with Koch's abdomen and received tuberculosis treatment for 1 year. However, she had persistent and worsening of symptoms, so a right-sided intercostal drainage tube was placed for a right pleural effusion. Image-guided pleural biopsy was performed and histopathology was suggestive of poorly differentiated carcinoma which was immunopositive for $\mathrm{CK} 7+$, ER+ and focal CA125+. The patient then self-referred to our hospital. After thorough work-up, she was planned for weekly TP neoadjuvant chemotherapy followed by surgical reassessment before proceeding with interval CRS with HITAC. Details of the surgical procedure were mentioned earlier and are based on pre- and intra-operative clinical findings. Post-operative histopathology was consistent with FIGO stage IVA. In the early postoperative period, the patient developed a recurrent right pleural effusion which necessitated another right intercostal drainage tube. After delayed clinical recovery, six cycles of adjuvant TP and bevacizumab were administered to the patient. The patient is currently alive with persistent disease at the last follow-up on 13-03-2020 and is still on bevacizumab based chemotherapy.

Patient serial number " 3 " is a 29 -year-old female with no known comorbidities, significant family history nor past medical or surgical history. She presented with a dry cough and shortness of breath for 10 months. The patient was worked-up and diagnosed with ovarian carcinoma with right sided malignant pleural effusion (FIGO IVA). Multidisciplinary tumor board discussion advised for TP based neoadjuvant chemotherapy followed by CRS and intraperitoneal/intrathoracic chemotherapy. Post 3 cycles TP, the patient underwent interval CRS with HITAC. The procedure details are mentioned in Table 1 and the postoperative period was uneventful. Final histopathology reported the same FIGO stage disease because of similar tumor deposits on the pleura. Three cycles of adjuvant TP regimen were administered to the patient. At the last follow-up on 19-09-2019, the patient was alive and disease-free.

\section{CONCLUSION}

CRS with HITAC is a complex and evolving procedure. These are viable treatment options for cases of ovarian carcinoma with peritoneal carcinomatosis and pleural disease in the post neoadjuvant chemotherapy setting. Macroscopic disease can be removed with CRS and the remaining microscopic disease can be dealt with through HITAC to reduce thoracic recurrences. In this study, there were no lifethreatening surgical morbidities. No mortality was recorded till the last follow-up. Following the technique described in this study, CRS with HITAC can be safely performed and replicated easily without additional morbidity or need for extra resources for HITAC. However, multicenter studies with larger numbers of patients and longer follow-up is warranted to establish reproducibility and acceptance of the procedure.

\section{DECLARATIONS}

\section{Acknowledgments}

We like to acknowledge our head of department Professor (Dr.) S V S Deo, DR BRA-IRCH, AIIMS, New Delhi for constant support and inspiration. The acknowledgment extends to all faculty and senior residents of the Department of Surgical Oncology and Department of Onco-anesthesia and Palliative Care for intraoperative and postoperative patient care. 


\section{Authors' contributions}

Substantial contributions to the conception or design of the manuscript, writing manuscript, revising it critically for important intellectual content and to be accountable for all aspects of the work in ensuring that questions related to the accuracy or integrity of the manuscript: Ray MD

Data collection, data analysis, data curation, drafting the work, manuscript writing, editing, conceptualisation, technical and material support, study supervision, revising it critically for important intellectual content and proofreading with final approval of the manuscript version to be published: Kumar $\mathrm{N}$

Data collection, data analysis, drafting the work, manuscript writing, drawing pictorial depiction and formatting, editing, provided technical, and material support: Kuppusamy $\mathrm{R}$

Manuscript writing and final approval of manuscript version to be published: Garg R

Have read and approved the manuscript: Ray MD, Kuppusamy R, Kumar N, Garg R

\section{Availability of data and materials}

The data (operative pictures, further case details, follow up data) to support the findings of this study are available on request from the corresponding authors.

\section{Financial support and sponsorship}

None.

\section{Conflicts of interest}

All authors declared that there are no conflicts of interest.

\section{Ethical approval and consent to participate}

The study has been performed following the "Declaration of Helsinki" and approved by the "institutional ethics committee, AIIMS, New Delhi, India" vide reference no: IEC-592/03.11.2017, AA-3/29. Informed consent to participate in the study has been obtained from participants.

\section{Consent for publication}

Not applicable. No identifying images or other personal or clinical details of participants are presented in the manuscript that compromise anonymity.

\section{Copyright}

(C) The Author(s) 2020.

\section{REFERENCES}

1. Mulier S, Claes JP, Dierieck V, Amiel JO, Pahaut JP, et al. Survival benefit of adding Hyperthermic IntraPEritoneal Chemotherapy (HIPEC) at the different time-points of treatment of ovarian cancer: review of evidence. Curr Pharm Des 2012;18:3793-803.

2. Chua TC, Yan TD, Saxena A, Morris DL. Should the treatment of peritoneal carcinomatosis by cytoreductive surgery and hyperthermic intraperitoneal chemotherapy still be regarded as a highly morbid procedure?: a systematic review of morbidity and mortality. Ann Surg 2009;249:900-7.

3. Sugarbaker PH. Peritonectomy procedures. Cancer Treat Res 2007;134:247-64.

4. Dehal A, Smith JJ, Nash GM. Cytoreductive surgery and intraperitoneal chemotherapy: an evidence-based review-past, present and future. J Gastrointest Oncol 2016;7:143-57.

5. Overgaard J. Effect of hyperthermia on malignant cells in vivo. A review and a hypothesis. Cancer 1977;39:2637-46.

6. van Driel WJ, Koole SN, Sikorska K, Schagen van Leeuwen JH, Schreuder HWR, et al. Hyperthermic intraperitoneal chemotherapy in ovarian cancer. N Engl J Med 2018;378:230-40.

7. Spiliotis J, Halkia E, Lianos E, Kalantzi N, Grivas A, et al. Cytoreductive surgery and HIPEC in recurrent epithelial ovarian cancer: a prospective randomized phase III study. Ann Surg Oncol 2015;22:1570-5.

8. Sugarbaker PH, Chang D, Stuart OA. Hyperthermic intraoperative thoracoabdominal chemotherapy. Gastroenterol Res Pract 2012;2012:623417.

9. Erasmus JJ, Goodman PC, Patz EF Jr. Management of malignant pleural effusions and pneumothorax. Radiol Clin North Am $2000 ; 38: 375-83$. 